\title{
Genotype 4 Hepatitis E Virus Prevalent in Eastern China Shows Diverse Subtypes
}

\author{
Hua Tian ${ }^{1}$; Xingli Fu ${ }^{2}$; Wang Li $^{2}$; Yuan Huang ${ }^{2}$; Jiayao Sun ${ }^{2}$; Gai Zhou ${ }^{2}$; Chengli Zhou ${ }^{2}$; \\ Quan Shen ${ }^{2}$; Shixing Yang ${ }^{2}$; Wen Zhang ${ }^{2, *}$ \\ ${ }_{1}^{1}$ Jiangsu Provincial Center for Disease Prevention and Control, Nanjing, China \\ 2 Department of Microbiology, School of Medicine, Jiangsu University, Zhenjiang, China \\ ${ }^{*}$ Corresponding Author: Wen Zhang, Department of Microbiology, School of Medicine, Jiangsu University, Zhenjiang, China. Tel: +86-51188785938, Fax: +86-51185038449, \\ E-mail: z0216wen@yahoo.com
}

Received: November 15, 2014; Revised: April 18, 2015; Accepted: June 6, 2015

\begin{abstract}
Background: Hepatitis E Virus (HEV), a zoonotic pathogen, uses several species of animal as reservoirs. Swine is considered as the major reservoir for HEV infection in humans. Genotype $4 \mathrm{HEV}$ is the dominant cause of hepatitis E disease in humans in China.

Objectives: Although many researches revealed that genotype $4 \mathrm{HEV}$ is the main genotype that prevalent in eastern China, few researches have done to study the subtype of HEV in this area. Thus, this study aimed to investigate the subtype of HEV prevalent in eastern China. Materials and Methods: A total of 125 anti-HEV IgM positive human serum and 290 swine fecal samples were subjected to reverse transcription polymerase chain reaction (RT-PCR) screening of HEV RNA. Positive PCR products were sequenced and phylogenetically analyzed.

Results: From a total of 125 human serum samples, 19.2\% (24.125) were positive, while 9.66\% (28.290) of the 290 swine fecal samples were positive for HEV RNA. Phylogenetic analysis based on partial capsid gene showed that the $51 \mathrm{HEV}$ strains in the current study all belonged to genotype 4, clustering into 6 different subtypes. Our results also revealed that some of HEV isolates prevalent in the human and swine populations were classified into the same clusters.

Conclusions: Genotype 4 HEV in eastern China shows subtype diversity and some HEV isolates are involved in cross-species transmission.
\end{abstract}

Keywords: Hepatitis E Virus; Genotype; Subtype; Genetic Diversity

\section{Background}

Hepatitis E virus (HEV), a member of the genus Hepevirus, is a non-enveloped virus with a positive-stranded RNA genome approximately $7.2 \mathrm{~kb}$ in length. HEV has been the cause of waterborne outbreaks of hepatitis in Asia and Africa and is a major cause of sporadic hepatitis in these regions. Acute HEV infection primarily affects young adults and is generally self-limiting and resolves in 1 - 6 weeks, except in women during late pregnancy, among whom $20 \%$ mortality has been reported; chronic HEV infection has recently been reported in transplant recipients (1). It has been hypothesized that zoonosis is involved in the transmission of $\operatorname{HEV}(2)$.

Hepatitis E virus and antibodies to HEV have been found in a wide variety of animals (3-6). Hepatitis E viruses were divided into 4 distinct genotypes: genotype $1,2,3$, and 4 . Genotypes 1 and 2 have been identified exclusively in humans, while genotypes 3 and 4 have been found in humans and several species of animals. Genotypes 1 and 2 have been isolated in Asia, Africa, and America; genotype 4 has been identified only in Asia; genotype 3 has been found almost worldwide $(7,8)$. Since 2000, genotype $4 \mathrm{HEV}$ has become the dominant cause of hepatitis E disease in China (9,10). Genotype 4 was proposed to be further classified into 24 subtypes (11). In the present study, we identified 52 genotype 4 hepatitis E viruses to subtype the HEV strains in eastern China. The relationship between HEVs from swine and human populations was also investigated.

\section{Objectives}

In the present study, we aimed to investigate the subtype of HEV prevalent in eastern China, and phylogenetically analyze the relationship between different HEV isolates prevalent in this area.

\section{Materials and Methods}

\subsection{Samples}

A total of 125 human serum samples, which were tested to be positive for anti-HEV IgM using commercial ELISAs (Wan Tai Pharmaceutical, China), were collected from local Centers for Disease Control and prevention (CDC) in Jiangsu Province by Jiangsu CDC from 2010 to 2013. A total

Copyright (C) 2015, Kowsar Corp. This is an open-access article distributed under the terms of the Creative Commons Attribution-NonCommercial 4.0 International License (http://creativecommons.org/licenses/by-nc/4.0/) which permits copy and redistribute the material just in noncommercial usages, provided the original work is properly cited. 
Tian $H$ et al.

of 290 swine fecal samples were obtained from pigs (aged 8 to 26 weeks old) in Zhenjiang City ( 80 samples) of Jiangsu Province, Anhui Province (90 samples) and Shanghai City (120 samples) from 2010 to 2012. Anhui Province and Shanghai City are two neighboring areas of Jiangsu Province. The three areas have the sample culture and interregional mobility of people is very common.

\subsection{Detection of Hepatitis E Virus-RNA}

All the fecal samples were converted to $10 \%$ (W/V) suspensions in 0.01 M Phosphate Buffered Saline (PBS) (pH 7.2 - 7.4). Fecal sample suspensions were then clarified by centrifugation at 10,000 $\mathrm{g}$ for 10 minutes and $100 \mu \mathrm{L}$ aliquots of the clarified material was used for viral RNA extraction. Total RNA was extracted from $100 \mu \mathrm{L}$ of serum sample or clarified fecal suspension, using Trizol (Invitrogen, USA). The viral RNA was finally dissolved in $20 \mu \mathrm{L}$ RNase-free water. Reverse transcription was performed using the TaKaRa RNA polymerase chain reaction (PCR) kit (TaKaRa, Japan) according to the manufacturer's protocol. Polymerase chain reaction was performed as described elsewhere (12). Briefly, a 348 bp segment of open reading frame 2 (ORF2), was amplified using primers E1 (5'-AATTATGCYCAGTAYCGRGTTG-3') and E2 (5'-CCCTTRTCYTGCTGMGCATTCTC-3') for the first round of PCR and primers E3 (5'-GTWATGCTYTGCATWCATGGCT-3') and E4 (5'-AGCCGACGAAATCAATTCTGTC-3') for the second round. The PCR cycling conditions for both rounds consisted of 35 cycles of denaturation for 30 seconds at $94^{\circ} \mathrm{C}$, annealing for 30 seconds at $50^{\circ} \mathrm{C}$, and extension for 40 seconds at $72^{\circ} \mathrm{C}$. The PCR products were analyzed in a $1.5 \%$ agarose gel. The expected DNA band specific for the HEV was excised from the gel, purified with the AxyPrep DNA gel extraction kit (Axygen, USA) and cloned into a pMD T-vector (TaKaRa, Japan). Both strands of the inserted DNA amplicons were sequenced in a DNA analyzer (Applied Biosystems 3730 DNA Analyzer; Invitrogen, USA).

\subsection{Phylogenetic Analysis}

The viral nucleotide sequences were aligned using ClustaLW2.0. Phylogenetic trees were constructed using the neighbor-joining method and evaluated using the interior branch test method with MEGA software version 5.0. Prototype HEV strains used as references in the analysis and their GenBank accession numbers are shown in the phylogenetic tree.

\section{Results}

Our results indicated that $19.2 \%$ (24.125) of the serum samples were positive for HEV RNA, while 9.7\% (28.290) of the swine fecal samples were positive for HEV RNA. Sequence analysis based on the PCR-amplified products (primer sequences were removed) indicated that the 52 HEV strains, including 24 from humans and 28 from pigs, all belonged to genotype $4 \mathrm{HEV}$, which suggested that genotype 4 was the main genotype prevalent in hu- man and swine population in this area during our sample collecting time. The 52 genotype $4 \mathrm{HEV}$ sequences shared more than $81.5 \%$ - $100 \%$ sequence identities and showed 49 distinct nucleotide sequences. In order to identify the subtype of the 49 genotype 4 HEV strains, phylogenetic analysis was performed based on the nucleotide sequences in the present study and other referenced HEV strains retrieved from GenBank and results were shown in Figure 1. Other 20 well-characterized subtypes of genotype $4 \mathrm{HEVs}$ in a previous report (11) were also included in the phylogenetic analysis, where the GenBank accession were not marked with black or squares. Results indicated that the 49 genotype $4 \mathrm{HEV}$ sequences in the present study were divided into 6 different subtypes, belonging to subtypes $4 \mathrm{~b}, 4 \mathrm{a}, 4 \mathrm{c}, 4 \mathrm{e}, 4 \mathrm{~g}$ and a new subtype, respectively.

Twenty-one of the 49 (42.9\%) genotype 4 strains were subtyped as $4 \mathrm{~b}$ and shared $92.5 \%$ - 99.1\% sequence similarity among themselves. The 21 subtype $4 \mathrm{~b}$ strains included 12 strains from pigs and 9 strains from human populations. Eight strains in the present study felt into the cluster of subtype 4 a, and shared $91.1 \%$ - 98.6\% identities among themselves, including four strains from humans and four strains from pigs. The subtype $4 \mathrm{c}$ included eight strains from the present study, which shared $87.8 \%$ - 99.0\% sequence identities among themselves, including six human originated and swine originated strains. Interestingly, four strains in this study could not be subtyped based on the defined subtypes, but closely clustered with several other strains in GenBank, forming a new subtype, sharing 92.2\% - 95.4\% sequence identities among themselves. Three strains including two strains from humans and one strain from pig clustered closely together and were subtyped into $4 \mathrm{e}$. The last three strains were all from swine populations and typed into subtype $4 \mathrm{~g}$.

\section{Discussion}

An increasing number of evidences indicate that HEV is enzootic and pigs were considered as the major reservoir for HEV infections in human populations $(9,13,14)$. The zoonotic transmission of HEV from pigs to humans has been suggested all over the world. Genotype 4 HEV was the main cause of hepatitis $\mathrm{E}$ in China and involved in zoonotic transmission in several areas of China $(13,14)$. The HEV isolates identified from the same geographic region tended to cluster together $(15,16)$. However, recently several studies suggested the HEV strain from a certain limited region may have several different subtypes ( 9 , 17). In the current study, we identified 49 genotype 4 HEV strains and phylogenetic analysis showed these isolates could be divided into six different subtypes, among which five subtypes contain isolates from both humans and pigs and the isolates from human and swine population closely clustered together, suggesting these five subtypes of genotype $4 \mathrm{HEV}$ were involved in cross-infection between human and pigs in this area. 


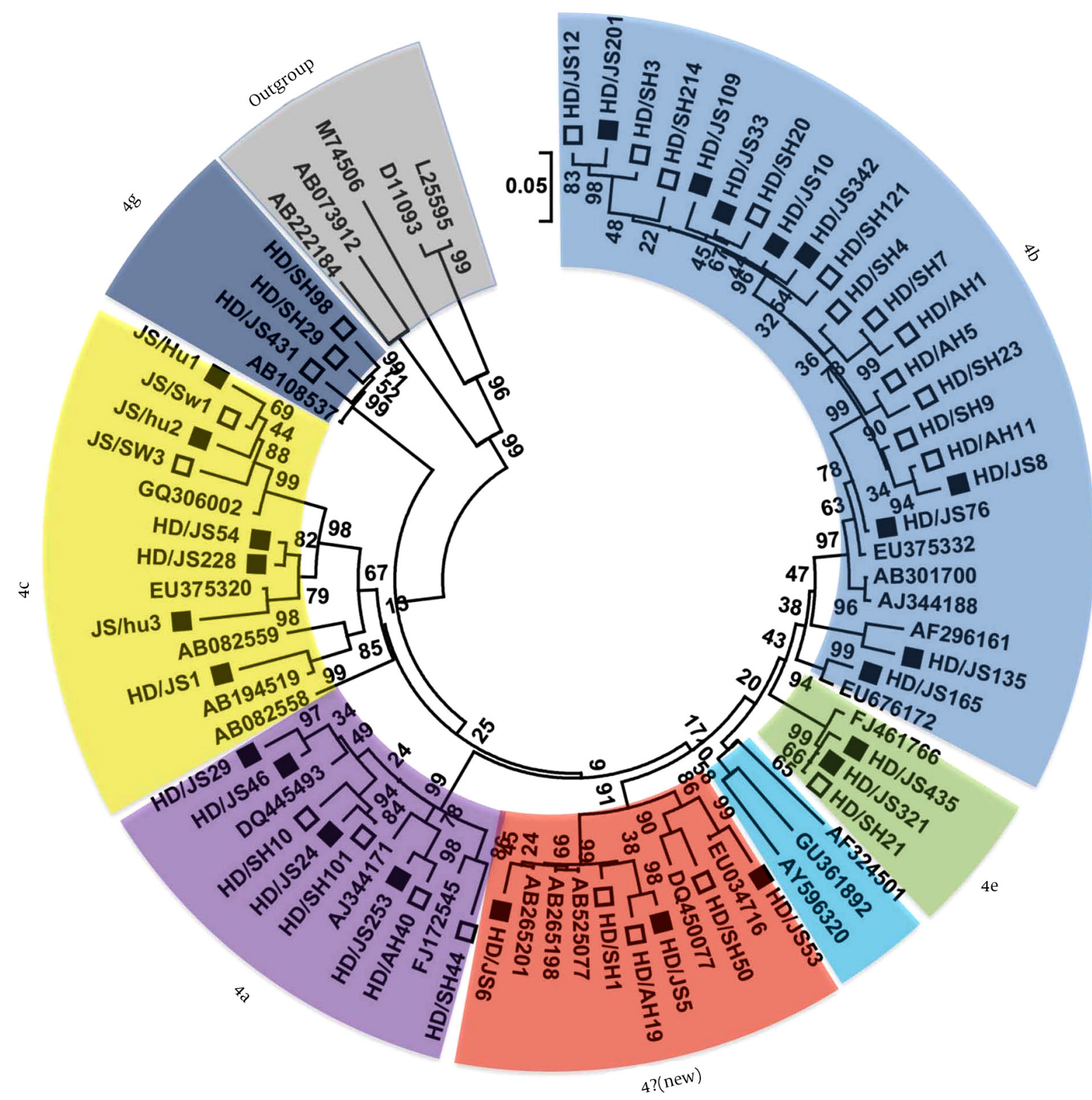

The phylogenetic tree was produced with a 348 bp ORF2 sequence alignments of 49 strains in the present study and the reference sequences from GenBank, using the neighbor-joining method and evaluated using the interior branch test method with Mega 5 software. The percent bootstrap support is shown by values at the branch nodes of the tree. These values were the result of resampling the data 1000 times. The isolates identified in the current study were marked with black or squares. The white squares indicated the sequences from pigs and the black indicated the sequences from humans.

The first swine strain of HEV was isolated and characterized from a pig in the United States (2). Subsequently, many HEV samples from swine in over a dozen countries have been identified. In the present study, although we did not classify the swine fecal samples according to the pig age because of the label on part of the collection plastic bags were missing unexpectedly, the overall positive rate of HEV RNA (9.66\%) was in agreement with several previously reports in other areas of China $(13,18)$. Hepatitis E virus isolates from human or pigs were divided into four distinct genotypes according to sequence and phylogenetic analyses. Recent several reports indicated that genotype 3 HEV was prevalent in swine and human populations in several regions of China $(19,20)$. However, the HEV strains in the present study all belonged to genotype 4 , which may owe to the different sampling site and time. 
In the present study, $42.9 \%$ (21.49) of the genotype 4 strains belonged to subtype $4 \mathrm{~b}$, including 12 strains from swine population and 9 strains from human, which suggested that subtype $4 \mathrm{~b}$ is the main subtype prevalent in swine and human populations in this area. Phylogenetic analysis also revealed that the subtype $4 \mathrm{~b}$ in the present study clustered with several other genotype 4 HEVs isolated from eastern China, while the other subtypes in this study clustered with HEVs from other regions in China or other countries, suggesting subtype $4 \mathrm{~b}$ HEVs are indigenous subtype of eastern China while the other subtypes may not be indigenous to this area.

\section{Acknowledgements}

The authors would like to thank for the financial supports from the National Natural Science Foundation of China No. 31302107, the Natural Science Foundation of Jiangsu Province, no. BK2012289, the Special Fund for Preventive Medicine of Jiangsu Province no. Y2012067, and Jiangsu university research project for undergraduate no. 12A125, 12A126, and 12A193.

\section{Authors' Contributions}

Hua Tian and Wen Zhang conceived the study. Hua Tian, Xingli Fu, Wang Li, Yuan Huang, and Jiayao Sun performed all the experiments. Gai Zhou, Chengli Zhou, Quan Shen and Shixing Yang performed the sequence and phylogenetic analysis. Hua Tian and Wen Zhang wrote the paper. All authors read and approved the final manuscript.

\section{Funding/Support}

This work was partly supported by the National Natural Science Foundation of China no. 31302107, the Natural Science Foundation of Jiangsu Province, no. BK2012289, the Special Fund for Preventive Medicine of Jiangsu Province no. Y2012067, and Jiangsu University Research Project for Undergraduate no.12A125, 12A126, and 12A193.

\section{References}

1. Kamar N, Selves J, Mansuy JM, Ouezzani L, Peron JM, Guitard J, et al. Hepatitis E virus and chronic hepatitis in organ-transplant recipients. NEngl J Med. 2008;358(8):811-7.

2. Meng XJ, Purcell RH, Halbur PG, Lehman JR, Webb DM, Tsareva TS, et al. A novel virus in swine is closely related to the human hepatitis E virus. Proc Natl Acad Sci U S A. 1997;94(18):9860-5.
3. Zhang W, Shen Q, Mou J, Gong G, Yang Z, Cui L, et al. Hepatitis E virus infection among domestic animals in eastern China. Zoonoses Public Health. 2008;55(6):291-8.

4. Zhou C, Li W, Yang S. Analysis of hepatitis e virus-like sequence in chimpanzee. Hepat Mon. 2014;14(9):e19473.

5. Han J, Zeng H, Wang L, Liu P, Liu L, Xia J, et al. Hepatitis E virus infection in farmed rabbits and swine in the Eastern Chinese city Lianyungang: showing no potential interspecies transmission. Med Virol. 2014;86(11):1898-904.

6. Widen F, Ayral F, Artois M, Olofson AS, Lin J. PCR detection and analysis of potentially zoonotic Hepatitis E virus in French rats. Virol J. 2014;11:90

7. Song YJ, Park WJ, Park BJ, Lee JB, Park SY, Song CS, et al. Hepatitis $\mathrm{E}$ virus infections in humans and animals. Clin Exp Vaccine Res. 2014;3(1):29-36.

8. Holla RP, Ahmad I, Ahmad Z, Jameel S. Molecular virology of hepatitis E virus. Semin Liver Dis. 2013;33(1):3-14.

9. Zhang W, He Y, Wang H, Shen Q, Cui L, Wang X, et al. Hepatitis E virus genotype diversity in eastern China. Emerg Infect Dis. 2010;16(10):1630-2.

10. Dai X, Dong C, Zhou Z, Liang J, Dong M, Yang Y, et al. Hepatitis E virus genotype 4, Nanjing, China, 2001-2011. Emerg Infect Dis. 2013;19(9):1528-30.

11. Lu L, Li C, Hagedorn CH. Phylogenetic analysis of global hepatitis E virus sequences: genetic diversity, subtypes and zoonosis. Rev Med Virol. 2006;16(1):5-36.

12. Zhang W, Shen Q, Mou J, Yang ZB, Yuan CL, Cui L, et al. Cross-species infection of hepatitis E virus in a zoo-like location, including birds. Epidemiol Infect. 2008;136(8):1020-6.

13. Zheng Y, Ge S, Zhang J, Guo Q, Ng MH, Wang F, et al. Swine as a principal reservoir of hepatitis E virus that infects humans in eastern China. J Infect Dis. 2006;193(12):1643-9.

14. Wang H, He Y, Shen Q, Wang X, Yang S, Cui L, et al. Complete genome sequence of the genotype 4 hepatitis $\mathrm{E}$ virus strain prevalent in swine in Jiangsu Province, China, reveals a close relationship with that from the human population in this area. J Virol. 2012;86(15):8334-5.

15. Huang FF, Haqshenas G, Guenette DK, Halbur PG, Schommer SK, Pierson FW, et al. Detection by reverse transcription-PCR and genetic characterization of field isolates of swine hepatitis E virus from pigs in different geographic regions of the United States. $J$ Clin Microbiol. 2002;40(4):1326-32.

16. Wibawa ID, Suryadarma IG, Tsuda F, Matsumoto Y, Ninomiya M Mulyanto., et al. Identification of genotype 4 hepatitis E virus strains from a patient with acute hepatitis $\mathrm{E}$ and farm pigs in Bali, Indonesia. J Med Virol. 2007;79(8):1138-46.

17. Yan Y, Zhang W, Shen Q, Cui L, Hua X. Prevalence of four different subgenotypes of genotype 4 hepatitis E virus among swine in the Shanghai area of China. Acta Vet Scand. 2008;50:12.

18. Zhang W, Yang S, Ren L, Shen Q, Cui L, Fan K, et al. Hepatitis E virus infection in central China reveals no evidence of cross-species transmission between human and swine in this area. PLoS One. 2009;4(12):e8156.

19. Geng Y, Zhao C, Fan J, Harrison TJ, Zhang H, Lian H, et al. Genotype analysis of hepatitis E virus from sporadic hepatitis E cases in northern China. Infect Genet Evol. 2013;20:413-7.

20. Zhang W, Yang S, Shen Q, Huang F, Shan T, Yang Z, et al. Genotype 3 hepatitis E virus existed among swine groups in 4 geographically far regions in China. Vet Microbiol. 2010;140(1-2):193-5. 